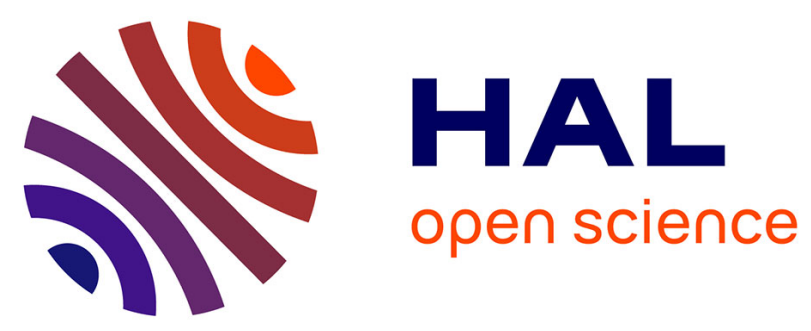

\title{
Information des couples et diagnostic prénatal d'une malformation cérébrale à pronostic incertain : analyse des pratiques
}

Grégoire Moutel, Marie-Laure Moutard, Irène François, Josué Feingold, Gérard Ponsot, Christian Hervé

\section{To cite this version:}

Grégoire Moutel, Marie-Laure Moutard, Irène François, Josué Feingold, Gérard Ponsot, et al.. Information des couples et diagnostic prénatal d'une malformation cérébrale à pronostic incertain: analyse des pratiques. Archives de Pédiatrie, 2004, 11 (5), pp.423-428. inserm-00118870

\section{HAL Id: inserm-00118870 https://www.hal.inserm.fr/inserm-00118870}

Submitted on 7 Dec 2006

HAL is a multi-disciplinary open access archive for the deposit and dissemination of scientific research documents, whether they are published or not. The documents may come from teaching and research institutions in France or abroad, or from public or private research centers.
L'archive ouverte pluridisciplinaire HAL, est destinée au dépôt et à la diffusion de documents scientifiques de niveau recherche, publiés ou non, émanant des établissements d'enseignement et de recherche français ou étrangers, des laboratoires publics ou privés. 


\title{
Information des couples et diagnostic prénatal d'une malformation
} cérébrale à pronostic incertain : analyse des pratiques

\author{
${\text { Grégoire } \text { Moutel }^{1} \text {,Marie-Laure Moutard }}^{1,2}$, \\ Irène François ${ }^{1,3}$, Josué Feingold ${ }^{1,2}$, \\ Gérard Ponsot $^{2}$, Christian Hervé ${ }^{1}$
}

\footnotetext{
${ }^{1}$ Laboratoire d'Ethique Médicale, de Droit de la Santé et de Santé Publique, IIREB (Institut International de Recherche en éthique biomédicale), Faculté de Médecine Necker, Université Paris V.

${ }^{2}$ Service de Neuropédiatrie, Hôpital saint Vincent de Paul

${ }^{3}$ Service de Médecine, Légale, Centre Hospitalo-Universitaire de Dijon, Université de Bourgogne.
} 


\title{
Information des couples et diagnostic prénatal d'une malformation cérébrale à pronostic incertain : analyse des pratiques
}

\begin{abstract}
Résumé
L'échographie fœtale (EF) est un examen très répandu, devenu quasiment systématique dans l'usage et élément majeur du dépistage des malformations foetales. L'annonce d'une malformation notamment cérébrale est particulièrement délicate car pèse souvent une double incertitude diagnostic et pronostic.

L'objectif de ce travail a été d'analyser comment s'organisent les pratiques en matière de DPN par imagerie et comment est réalisée la prise en charge et l'accompagnement des couples. Elle a particulièrement porté sur les procédures d'information : contenu, condition de la délivrance, et conséquences.

Une enquête multicentrique a été menée auprès des grands centres pluridisciplinaires de Paris et de sa région, par entretiens semi-directifs au moyen d'un questionnaire. Nous montrons les difficultés d'harmonisation concernant la délivrance de l'information avant et après l'examen, le risque d'induire davantage le recours à l'interruption médicale de grossesse lorsqu'il existe une incertitude sur le pronostic.

Ces résultats amènent à proposer des pistes de réflexion sur un parcours harmonisé permettant aux couples de bénéficier de toutes les compétences en matière de DPN, notamment en cas d'incertitude diagnostique et pronostique, et de la nécessité d'un réseau pluridisciplinaire de soutien et d'accompagnement à la décision, interruption ou conservation de la grossesse.
\end{abstract}

\section{Summary}

Fetal ultrasounds (FU) allow identification of brain malformations; announce of diagnosis and information about prognosis may be difficult when malformation is rare and prognosis uncertain.

The aim of this study was to analyze how imaging for prenatal screening is organized and how couples are managed and supported. We concentrated on the procedures used to inform couples: content, method of delivery and consequences.

Study amongst large multidisciplinary centers in Paris and the Paris region, by semi-directed interviews using a questionnaire.

We showed that it is difficult to standardize the way in which information is supplied before and after the FU; pediatricians (neuropediatrician) are not systematically involved in providing information; uncertainty about prognosis leads more often to abortion.

There is a need for multidisciplinary teams including pediatricians to inform, support parents, and to accompany their decision concerning pregnancy.

Mots-clés : diagnostic prénatal, information, malformation cérébrale, échographie fœetale. 


\section{Introduction}

Le diagnostic prénatal, proposé aux femmes pendant la grossesse, bien que non obligatoire, connaît depuis une vingtaine d'années un développement exponentiel. Il repose sur les tests à visée génétique et sur l'imagerie fœtale (échographie et résonance magnétique). Contrairement à l'imagerie fœtale, les tests à visée génétique (marqueurs sériques, biologie moléculaire, caryotype) occupent une place bien codifiée. Ils s'adressent en règle à une population restreinte et ciblée, femmes ou couples à risque (existence d'une pathologie génétique familiale, antécédent d'anomalie chromosomique, risque de trisomie 21 dépistés par HCG ou alphafoetoproteine) (1) ; ils sont encadrés par la législation puisque réalisés dans des laboratoires agréés et imposent le recueil d'un consentement éclairé après information (articles L2131 à 2131-5 du Code de Santé Publique) (2). En revanche, l'échographie foetale (EF), alors qu'il s'agit d'un examen très répandu, devenu quasiment systématique dans l'usage et élément majeur du dépistage des malformations fœetales notamment cérébrales, ne bénéficie pas d'un encadrement comparable. Bien que non obligatoire elle est d'un accès large et proposée à toute femme enceinte. Elle n'est pas encadrée par une législation, et les procédures d'information et de consentement des femmes (couples) ne sont pas clairement établis. Il s'agit d'un examen simple, reproductible, sans danger pour la mère et le fœetus (3), réalisé au moins trois fois au cours de la grossesse : à 12 semaines d'aménorrhée (SA), à 22 SA où l'étude morphologique de l'ensemble des organes est possible, et à 32 SA (4-6). Lorsqu'une anomalie est dépistée, l'EF peut être complétée par une imagerie par résonance magnétique nucléaire du cerveau fœtal $(\operatorname{IRM})(7,8)$ mais elle reste le premier et le plus performant examen de dépistage (9). 
Les couples sont souvent peu préparés à l'annonce d'une malformation, l'EF étant davantage vécue comme un examen permettant les premiers contacts avec l'enfant, l'appréciation de son bien être et non comme un moyen d'investigation médical visant à dépister des anomalies.

Ceci s'illustre dans le domaine des malformations cérébrales fœtales où l'annonce est particulièrement délicate et où pèse souvent une double incertitude diagnostic et pronostic.

Parmi les anomalies cérébrales accessibles au dépistage échographique, toutes n'ont pas le même pronostic et l'information donnée revêt donc une particulière importance puisqu'elle va conduire à une décision concernant la poursuite ou non de la grossesse.

Plusieurs situations se rencontrent en pratique :

- certaines malformations sont considérées comme bénignes parce qu'habituellement asymptomatiques ou sans retentissement sur le développement psychomoteur de l'enfant (10).

- d'autres sont sévères comme l'anencéphalie et constamment associées à un pronostic très péjoratif : enfant grabataire, peu ou pas d'acquisitions, épilepsie réfractaire (11).

- mais le cas le plus difficile est représenté par les malformations responsables d'expression clinique variable avec tantôt des individus asymptomatiques tantôt des individus présentant de manière plus ou moins associée et marquée des troubles cognitifs, des troubles du comportement ou une épilepsie : l'agénésie du corps calleux (ACC) fait partie de ces malformations à pronostic incertain et aucun signe prénatal ne permet de prévoir le phénotype de l'enfant à venir $(12,13)$.

Lorsqu'une malformation cérébrale sévère ou à pronostic incertain est dépistée, une interruption médicale de grossesse (IMG) peut être demandée. Il apparaît donc essentiel d'analyser les conditions de réalisation des EF et d'information des couples puisqu'elles conditionnent la prise décision. 
Nous avons donc étudié comment s'organisent les pratiques en matière de DPN par imagerie et comment est réalisée la prise en charge et l'accompagnement des couples.

Notre étude porte particulièrement sur les procédures d'information dans le cas de la plus fréquente des malformations cérébrales, l'ACC, procédures qui guident le choix éclairé des couples quant à l'issue de la grossesse.

Il s'agit d'une étude multicentrique reposant sur des entretiens semi-directifs par questions ouvertes posées oralement par l'investigateur. Ces entretiens ont été réalisés auprès de médecins gynécologues obstétriciens compétents en échographie fœtale pour analyser leur pratique en matière de diagnostic prénatal. Ce mode de recueil a été choisi parce qu'il permet d'avoir des réponses plus riches et plus complètes qu'un questionnaire écrit à questions simples adressé de manière anonyme. Les entretiens permettent en outre une description des pratiques et des jugements de professionnels impliqués dans le diagnostic prénatal de malformations neurologiques et dans l'accompagnement des couples à la décision. Le questionnaire a été testé auprès de médecins pour en évaluer la compréhensibilité et la faisabilité avant d'être mis en place définitivement auprès du panel choisi (encadré 1).

Trois grands thèmes ont été abordés :

- connaissances et opinions des professionnels sur les conditions de réalisation technique et l'encadrement de l'échographie fœtale (lois, chartes ou recommandations),

- information donnée aux couples concernant le diagnostic d'une malformation cérébrale, - information quant au pronostic et conséquences.

La région Ile de France concernée par notre étude représente 1/6 de la population française (11 millions d'habitants, correspondant à près de 236845 grossesses par an) et comporte 7 principaux centres pluridisciplinaires de diagnostic prénatal (CPDPN) auxquels vont obligatoirement avoir recours les couples en cas de DPN d'une malformation cérébrale. Dans 
chacun de ces centres, dont 6 ont accepté de participer à notre étude, un médecin référent a été choisi pour répondre au questionnaire. Par ailleurs le Président du Collège Français d'Echographie Fœtale (CFEF) représentant l'ensemble de la profession, et référent auprès des CPDPN a également été interrogé.

Au total 7 entretiens de référents ont été réalisés, enregistrés après accord du médecin et retranscrits de manière anonyme. L'analyse de contenu s'est faite à l'aide d'une grille de lecture analysant thèmes, mots clé, idées dominantes ainsi que la concordance et la divergence des réponses.

\section{Les questions en pratique}

Les 7 médecins interrogés (6 hommes et une femme) sont gynécologues-obstétriciens et compétents en EF ; leur âge moyen est de $48 \pm 7.8$ ans [36-57 ans] et la durée moyenne de leur pratique est de $18 \pm 9.8$ ans [5-30 ans].

\section{Conditions et opinions en EF}

L'ensemble des médecins considère que trois échographies sont indiquées au cours de la grossesse, une par trimestre, mais disent qu'aucune n'est obligatoire. Tous constatent qu'aucune législation n'encadre l'EF (et a fortiori l'IRM) mais signalent que des règles de bonne pratique ont été établies par diverses associations professionnelles : celles du CFEF et du Collège National des Gynécologues Obstétriciens Français (CNGOF) ont été citées (14, 15). Toutefois ces règles ne sont pas reconnues par tous : des recommandations sur le déroulement de l'examen («check list» des organes fœtaux à repérer et analyser), sur le 
contenu du compte rendu sont signalées par 3/7. Pour 4/7, il n'y a pas de guide précis sur les conditions de réalisation de l'examen et du rendu des résultats. Et il apparaît par ailleurs des divergences de point de vue entre les médecins interrogés quant à l'intérêt de telles procédures.

Le recours à l'IRM est loin de faire l'unanimité : certains pensent (2/7) qu'elle doit être réservée aux incertitudes diagnostiques, d'autres qu'elle sert à conforter un diagnostic (2/7). Dans un cas un médecin insiste sur le fait qu'elle représente une aide à la décision. Pour l'ensemble des personnes interrogées, l'indication de cet examen est du ressort de l'échographiste et non du médecin traitant gynécologue.

La population des échographistes fotaux est décrite par les référents des CPDPN comme hétérogène, hétérogénéité qui concerne tant la formation initiale (radiologue, gynécologue obstétricien, médecin généraliste, sage-femme) que la formation médicale (pas de diplôme obligatoire pour l'EF). Ceci explique que tous les échographistes n'aient pas le même rôle ni la même compétence. Les médecins questionnés parlent de niveau de compétence, de basse prévalence et d'examen de routine établissant ainsi une distinction entre les échographistes qu'ils classent par le niveau : il y a ceux de niveau 1, routine, simple dépistage, par opposition à ceux de niveau 2, plus compétents parce que capables de dépister les anomalies rares, parce qu'appartenant à des équipes pluridisciplinaires, ou 3, référents ou experts auxquels ils s'assimilent. 


\section{Information avant l'examen}

Cinq médecins sur 7 ne donnent qu'une information très limitée concernant le but de l'examen (examen de l'enfant, recherche d'anomalies), sans détailler le type d'anomalie recherchée ni les conséquences.

Les raisons invoquées sont multiples :

- Les médecins pensent que l'EF fait partie intégrante de la grossesse et que ses objectifs seraient connus des patientes.

- Les référents des CPDPN supposent que l'information sur le pourquoi de l'EF a déjà été donnée en amont par un autre praticien.

- Ils mettent en avant la difficulté de définir quelle information donner.

- Et ils soulignent notamment l'impossibilité d'être exhaustif dans la liste de ce qui va être recherché.

Deux médecins disent donner une information sur les buts, les limites et les conséquences de l'examen.

Que l'information donnée soit complète ou non, l'ensemble des médecins reconnaît qu'on devrait donner une information avant tout examen, faisant état de l'aspect médical et scientifique de l'EF, de sa finalité qui est la recherche de malformations foetales pouvant déboucher sur une IMG, mais aussi de ses limites, et de l'impossibilité d'annoncer tout ce qui va être recherché (risque de surinformation, d'angoisse). L'intérêt d'un document écrit remis à la patiente et expliquant tout ceci est mentionné par 4 médecins sur 7 . 


\section{Information sur le résultat de l'examen}

\section{1- Diagnostic}

Le questionnaire renseigne sur la personne qui donne le diagnostic d'ACC et sur les conditions de l'annonce.

Dans tous les cas ce sont les médecins qui font l'EF qui donnent le diagnostic et ils estiment que cette responsabilité leur incombe ; l'annonce n'est jamais laissée au gynécologue traitant, 2/7 disent le tenir au courant et un seul signale la possibilité d'inclure celui-ci dans l'équipe pluridisciplinaire qui va suivre la patiente après le diagnostic d'ACC.

L'annonce des résultats est faite en fin d'examen pour 5 médecins ; pour deux le diagnostic est évoqué en temps réel pendant l'examen.

Quel que soit le moment choisi, l'ensemble des référents disent l'importance du silence pendant l'examen en utilisant des termes comme «asepsie verbale», ou «mutisme bienveillant », témoin pour eux d'une image de compétence et permettant par ailleurs le recul si des anomalies sont retrouvées. L'importance de faire l'annonce à une patiente non allongée, rhabillée $(5 / 7)$ et dans une pièce différente de la pièce d'examen pour deux d'entre eux est soulignée. La présence des deux conjoints est fortement souhaitée pour 6 médecins sur 7, non obligatoire pour $1 / 7$.

\section{2- Pronostic}

Quatre référents disent la difficulté à établir un pronostic devant une ACC fœtale et le mot « incertitude » est cité par la totalité du panel interrogé. Deux donnent une information sur le pronostic, 5 préfèrent confier cette information à d'autres médecins considérés comme spécialistes de la malformation en cause (neuropédiatres) et intervenir ensuite comme «agent de synthèse ». L'information est modulée en fonction du vécu du couple pour $5 / 7$, identique 
pour 2 médecins : lorsqu'on est en présence d'un couple à risque (ayant déjà été confronté à un DPN positif), l'annonce est plus «ciselée » ou modulée pour prendre en compte le traumatisme antérieur.

Trois médecins évoquent la possibilité d'une IMG mais sans guider le choix de la patiente ou du couple; 4 ne l'évoquent pas dans les entretiens. Tous les référents pensent que l'information donnée tant sur le diagnostic que sur l'incertitude du pronostic, va plus souvent induire l'IMG que la poursuite de la grossesse.

En définitive si l'annonce du diagnostic est la prérogative de celui qui fait l'examen, les avis sont plus nuancés en ce qui concerne l'annonce du pronostic. Certains assument cette annonce, d'autres la délèguent et font appel au neuropédiatre. Tous reconnaissent qu'il s'agit d'une annonce difficile et qu'il n'y a pas de consensus sur le pronostic.

Cette incertitude bien souvent conduit à l'IMG.

\section{Gestion de l'incertitude et risque d'une approche normative}

Le DPN par imagerie fœtale est l'outil de dépistage des malformations fœtales. Il s'adresse à toutes les femmes, et bien que non obligatoire, il est devenu pratiquement systématique.

Les procédures de l'information dans ce domaine ne sont pas clairement définies et ceci pour de multiples raisons renvoyant à la complexité de l'information, à l'absence d'études permettant d'avoir des critères pronostiques fiables. Par ailleurs il n'y a pas de procédures définissant qui doit donner l'information et en fonction de quelles compétences.

Nos résultats renforcent l'idée de l'intérêt d'organiser un parcours codifié pour l'ensemble des couples qui soit harmonisé entre les différents centres; ceci permettrait que tout couple concerné puisse bénéficier réellement de toutes les compétences. Ceci permettrait en outre de 
recenser l'ensemble des cas avec des évaluations communes multidisciplinaires dans le but d'études épidémiologiques et d'amélioration des connaissances.

La collaboration pluridisciplinaire (gynécologues obstétriciens, échographistes fœtaux, neuropédiatres ayant l'expérience du suivi d'enfants nés avec cette malformation, généticiens, travailleurs sociaux en charge du handicap) est le moyen de mettre en place de telles filières de soins pour les couples confrontés à ce délicat problème et respecter ainsi le droit des patients : droit à l'information complète et éclairée, droit au double avis, droit à la collégialité dans la démarche de soins.

Face à une anomalie cérébrale, l'ACC, dont l'expression clinique est variable, où les avis convergent pour reconnaître la difficulté d'établir un pronostic, les professionnels sont amenés à partager cette incertitude. En l'absence d'arguments objectifs ou scientifiquement démontrés, ils vont réagir en fonction de leurs présupposés (16). Et le présupposé majeur dans ce domaine concerne l'acceptation familiale, sociale et le regard sur le handicap $(17,18)$. Le discours issu de ce vécu ne peut être neutre et toute intervention du médecin à travers l'information donnée, que ce soit avant l'EF ou après le diagnostic, influence le choix de la patiente ou du couple. Nos résultats montrent que les médecins ont pleinement conscience de leur poids dans la décision. Reste à savoir si, majoritairement, l'orientation du discours donné n'est pas univoque, induisant implicitement l'IMG, sous tendue par le refus du retard surtout intellectuel, du handicap quel qu'il soit chez l'enfant à venir.

Une autre dimension plus récente aggrave ce risque de discours «normatif »: la crainte de l'erreur et des poursuites en cas de handicap sévère et la recherche du risque zéro (19). En effet en France en juillet 2001, l'indemnisation d'un adulte lourdement handicapé a été prononcée au seul motif d'être né handicapé suite à une erreur médicale $(20,21)$.

L'information ne doit pas être conçue et pensée dans le cadre de l'urgence : même dans le temps limité d'une grossesse, il n'y a pas d'urgence à quelques semaines pour que les couples 
décident. L’information doit donc être la plus équilibrée et la plus complète possible, sans chercher à orienter la décision, pour permettre aux couples de s'approprier progressivement les concepts médicaux et leurs conséquences. C'est dire l'importance mais aussi la difficulté de définir des référentiels en matière d'information, de modalité de délivrance, tels que le souhaitent ou y ont réfléchi les professionnels à l'exemple du Collège français d'Echographie Fœtale, du Collège national des Gynécologues Obstétriciens Français ou de l'Agence Nationale d'Accréditation et d'Evaluation en Santé (ANAES) $(9,14,15)$.

\section{Conclusion}

Ces résultats amènent à discuter des principes qui pourraient aider les libres choix éclairés des patients :

-recours au deuxième avis d'un spécialiste référent, pédiatre, neuropédiatre impliqué dans le suivi et la prise en charge d'enfants porteurs de la malformation permettant un regard plus juste sur le handicap

-emploi d'un discours évitant de faire apparaître un lien obligatoire entre malformation et IMG (22)

-cohérence des différents intervenants au sein d'un parcours pluridisciplinaire pour constituer un réseau de conseil et de soutien autour du couple.

Le rôle du médecin, après ce temps partagé d'information et d'écoute est de conforter et soutenir le couple, l'accompagner dans son choix, quel que soit ce choix, respectant ainsi son autonomie sans pour autant le laisser à sa solitude. 


\section{Remerciements}

Aux Centres Pluridisciplinaires de Diagnostic Prénatal des hôpitaux Cochin saint Vincent de Paul (Paris), saint Antoine (Paris), Necker-Enfants malades (Paris), Institut de Puériculture (Paris), Antoine Béclère (Clamart), du Centre Hospitalier de Poissy, au Docteur Bessis (Paris), à l'Institut de Recherche en Ethique Biomédicale (IREB) qui a permis et soutenu la réalisation de ce travail. 


\section{Références}

1 - Code de Santé Publique : www.legifrance.org

2 - Cederholm M, Axelsson O, Sjoden PO. Women's knowledge, concerns and psychological reactions before undergoing an invasive procedure for prenatal karyotyping. Ultrasound Obstet Gynecol $1999 ; 14: 267-72$.

3- Barnett SB, Ter Haar GR, Ziskin MC, Rott HD, Duck FA, Maeda K. International recommendations and guidelines for the safe use of diagnosis ultrasound in medecine. Ultrasound Med Biol $2000 ; 26$ : 355-366.

4 - Chervenak FA, Mc Cullough LB. Ethical dimensions of ultrasound screening for fetal anomalies. Ann N Y Acad Sci 1998 ; 18 : 185-190.

5 - Collège National des Gynécologues et Obstétriciens Français. Apport de l'Echographie en Obstétrique. Conférence de Consensus Paris 2 et 3 décembre 1987. Arch Fr Pédiatr 1989; 46 : 307-312.

6 - Levi S. Routine ultrasounds screening of congenital anomalies. An overview of the European Experience. Ann N Y Acad Sci 1998 ; 13 : 86-98.

7 - Levine D, Barnes PD, Madsen JR, Abbott J, Mehta T, Edelman RR. Central nervous system abnormalities assessed with prenatal magnetic resonance imaging. Obstet Gynecol ; 1999: $94: 1011-9$.

8 - Whitby E, Paley MN, Davies N, Sprigg A, Griffiths PD. Ultrafast magnetic resonance imaging of central nervous system abnormalities in utero in the second and the third trimester of pregnancy : comparison with ultrasounds. BJOG $2001 ; 108: 519-26$.

9 - Agence National d'Accréditation et d'Evaluation en Santé (ANAES). L'échographie obstétricale au cours de la grossesse en l'absence de facteur de risque. Rapport. 1998, Service des Recommandations et Références professionnelles.

10 - Kinzler WL, Smulian JC, Mclean DA, Guzman ER, Vintzileos AM. Outcome of prenatally diagnosed mild unilateral ventriculomegaly. J Ultrasound Med $2001 ; 20: 257-61$.

11 - Alembik Y, Dott B, Roth MP, Stoll C. Prevalence of neural tube defects in Northeastern France, 1979-1992. Impact of prenatal diagnosis. Ann Génét $1995 ; 38: 49-53$.

12 - Moutard ML, Lewin F, Baron JM, Kieffer V, Descamps P. Prognosis of isolated agenesis of the corpus callosum. Neurochirurgie $1998 ; 44$ (1 Suppl) : 96-8.

13 - Moutard ML. Diagnostic prénatal des anomalies cérébrales : le doute peut-il être partagé ? Neurologies $2001 ; 4: 363-365$.

14 - Collège français d'Echographie Fœtale : www.cfef.org.

15 - Collège national des Gynécologues Obstétriciens Français : www.cngof.asso.fr. 
16 - Milliez J. L’Euthanasie du fœtus, médecine ou eugénisme ? Odile Jacob 1999.

17 - De Muylder X. Diagnostic anténatal et handicap : quelles logiques sociales. La presse Médicale $2001 ; 14: 684-5$.

18 - Detraux JJ, Gillot-de-Vries FR, Vanden Eynde S, Courtois A, Desmet A. Psychological impact of the announcement of a fetal abnormality on pregnant woman and on professionals. Ann N Y Acad Sci $1998 ; 13$ : 210-19.

19 - Moutel G, Hervé C. Les risques d'une application aveugle du principe de précaution en médecine. Press Med, 2001 ; 30 : 125-128.

20 - Arrêt Perruche, textes de lois :

Cassation Assemblée plénière., 17 novembre 2000 (Bull civ, n9) rapp. P. Sargos, concl. J. Sainte-Rose.

Cassation Assemblée plénière., arrêt n480, 13 juillet 2001.

Cassation Assemblée plénière., arrêt n479, 13 juillet 2001.

Cassation Assemblée plénière., arrêt n47, 13 juillet 2001.

21 - Moutel G, François I, Moutard M.L, Hervé C. L'arrêt Perruche : une occasion de nous interroger sur l'acceptation du handicap et sur les rapports entre médecine, justice et société. Presse médicale, accepté 02/2002 .

22 - Maroteaux P. Réflexions sur les problèmes éthiques du diagnostic prénatal. Arch Fr Pediatr $1984 ; 41: 445-8$. 


\section{Encadré 1: Questionnaire}

Profil professionnel

Diagnostic prénatal d'une malformation à pronostic incertain : l'agénésie isolée du corps calleux

-Tout d'abord nous souhaiterions avoir quelques renseignements concernant votre activité professionnelle

Q1 - Quelle est la spécialité sous laquelle vous être inscrit à l'ordre des médecins ?

Q2 - Quel pourcentage de votre activité représentent les échographies fœtales?

Q3 - Quelles autres types d'échographies pratiquez vous?

Q4 - Quel est le lieu principal où vous pratiquez ces échographies :

Q5 - Depuis combien de temps exercez vous cette activité d'échographie fœtale ?

Q6 - Quel âge avez vous?

Q7 - Avez vous des enfants ?

Q8 - Sexe

$>\quad$ Réalisation technique : état des lieux

Maintenant nous souhaiterions connaître comment s'organise la pratique de l'échographie fotale

Q9 - dans votre pratique

Pendant la grossesse, combien d'échographies jugez vous nécessaires?

A quel terme?

Q10 - Y a $t$ il une législation en matière d'échographie fotale ?

Q11 - Y a-t-il des règles ou chartes professionnelles en matière d'échographie fœtale ?

Q12 - Tous les échographistes ont-ils

le même rôle? oui non,

pourquoi

la même compétence? oui non

pourquoi

Q13 - Existe-t-il des référents ou des experts ?

Si oui, lesquels?

Qui ou quoi leur confère cette qualité ?

Q14 - Existe-t-il des Centres agréés d'échographie fœtale ?

Q15 - Y a t il pour vous une place pour l'IRM dans les malformations foetales

Quelles en sont pour vous les indications?

Qui doit la prescrire?

Q16 - Y a-t-il des règles ou chartes professionnelles en matière d'IRM fotale ?

$>\quad$ L'information donnée au couple

Venons-en à l'agénésie du Corps Calleux (ACC) ou absence de «pont » reliant les deux hémisphères cérébraux.

Q17 - Avant de réaliser l'examen :

A votre avis quelle information doit-on donner au couple sur la finalité (objectifs) de l'échographie ?

Quelle information donnez vous en pratique avant de faire cet examen ?

L'information que vous donnez est-elle différente selon dépistage systématique ou couple à risque ?

Q18 - Combien de fois avez-vous eu l'occasion de diagnostiquer une ACC à l'écho au cours de l'année 2000 ?

Q19-Combien y a-t-il eu d'IMG?

Q20 - Dans quel pourcentage selon vous cette malformation est dépistée lors d'un examen systématique ou lors d'un examen orienté ?

Q21 - Comment annoncez vous cette anomalie lorsque c'est vous qui la dépistez ?

Annoncez vous même le diagnostic?

Quand?

Ou renvoyez vous le couple au gynécologue ou médecin traitant à qui vous confiez l'annonce du diagnostic ?

Pensez vous que la mère et le père doivent être présents ensemble pour l'annonce du diagnostic ?

Q22- Comment annoncez-vous l'anomalie lorsque vous intervenez en $2^{\text {ème }}$ lieu ?

Annoncez vous même le diagnostic ? Quand?

Ou renvoyez vous le couple au gynécologue ou médecin traitant à qui vous confiez l'annonce du diagnostic ?

Pensez vous que la mère et le père doivent être présents ensemble pour l'annonce du diagnostic

Q23- Prescrivez vous des examens ou des consultations complémentaires si découverte d'une ACC ?

Une échographie de contrôle?

IRM fotale ?

Amniocentèse ?

consultation spécialiste, neuropédiatre ou neurologue, ou autre ?

le couple est-il envoyé à un centre pluridisciplinaire de dg prénatal ?

Q24 - Prescrivez vous ces mêmes examens ou consultations supplémentaires si le couple s'orientent vers une interruption de grossesse ?

Q25 - Y a-t-il un retentissement de cette malformation cérébrale sur le développement psycho-moteur de l'enfant après la naissance ?

Q26 - Que pensez vous du pronostic de l'ACC isolée ?

Q27 - Pensez vous qu'il y ait un consensus parmi les soignants sur le pronostic d'une ACC isolée ?

Q28 - Donnez vous une information au couple sur le pronostic de l'ACC isolée ?

Q29 - Un compte rendu écrit est-il établi dans tous les cas ?

Q30 - Y a t-il un document signé par la patiente ou le couple disant que l'information a été donnée ?

$>\quad$ La décision de poursuivre ou d'interrompre la grossesse

Q31 - Avec quel(s) intervenant(s) se discute la décision concernant la grossesse ?

Q32 - Pensez vous que la mère et le père doivent être présents ensemble pour décider ?

Q33 - Lorsque l'annonce d'une ACC a déjà été faite, que pensez vous de l'impact l'information donnée préalablement ?

Q34 - Dans combien de cas avez vous rectifié une erreur diagnostic

Dans le sens d'un anomalie bénigne ou pas d'anomalie (combien de fois)

Dans le sens d'une malformation plus sévère (combien de fois)

Commentez ces deux points : 
Q35 - Si vous donnez l'information au couple sur les résultats de l'échographie : avez-vous l'impression que l'information que vous avez donnée a :

a) influencé la décision du couple ?

b) modifié la décision que le couple avait prise avant de vous voir?

c)et à votre avis quel a été l'élément déterminant dans ce changement ? 